\title{
Assembly and Characterization of a Prototype Laser-Optical Firing System
}

\author{
Gregg L. Morelli*a \\ ${ }^{a}$ NNSA’s Kansas City Plant, 2000 E. $95^{\text {th }}$ St., Kansas City, MO, USA
}

\begin{abstract}
The design, assembly and characterization of the latest generation of a small, ruggedized laser-optical firing system will be discussed. This work builds upon earlier results in an effort to continue the development of robust fiber-coupled laseroptical firing systems. ${ }^{[1][2]}$ This newest prototype strives to improve on earlier designs, while continuing to utilize many of the environmentally proven opto-mechanical sub-assemblies. ${ }^{[2][3]}$ One area of improvement involves the implementation of a second optical safing and arming component. Several additional design improvements were also incorporated to address shortcomings uncovered during environmental testing. ${ }^{[4][5]}$ These tests and the subsequent failure analysis were performed at the laser sub-system level. Four identical prototypes were assembled and characterized. The performance of the units were evaluated by comparing a number of parameters including laser output energy, slope efficiency, beam divergence, spatial intensity profile, fiber injection and splitter-coupler transmission efficiency. Other factors evaluated were the ease of alignment, repeatability of the alignment process and the fabrication of the fiberoptical cables. The experimentally obtained results will be compared and contrasted to the performance of earlier prototypes.
\end{abstract}

Keywords: Solid-State Lasers, Fiber Optics, Beam Injection, Environmentally Rugged

\section{INTRODUCTION}

The goal of this project was to assemble and characterize the latest design iteration for a fiber-coupled laser firing system. Four 2008 Prototype units were assembled and characterized to obtain a sense of the assembly and performance repeatability of this design. This work builds upon earlier efforts to compare the performance of two possible design options, a "Diffractive" design and a "Baseline" design. ${ }^{[1]}$ It was concluded that the "Baseline" design was more viable than the "Diffractive" design, and as such provided the initial starting point for this latest prototype. A number of design improvements were incorporated with the goal of improving the overall system performance and ease of assembly. A rhomboid prism was incorporated as a second optical safing and arming component, the laser pump cavity was redesigned to improve the laser performance and improve the mechanical robustness. The core diameter of the fiber optical cables was decreased to facilitate cable routing and address minimum bend radius concerns. Additionally, the fiber optical cables were terminated with a high power version of the standard SMA 905 connector.

This work was the result of a highly collaborative on-going relationship between scientists and engineers at Sandia National Laboratories and Honeywell Federal Manufacturing and Technologies. The conceptual design activity, while primarily the responsibility the National Laboratory, was preformed with the considerable involvement of the production agency. This "Concurrent Engineering" model has proven to be a very effective method for reducing the development cycle time required for these prototype systems.

*gmorelli@kcp.com; phone 1816 997-7286; fax 1816 997-5614; kcp.com 


\section{BACKGROUND}

The 2008 Prototype system (Figure 1) utilizes a laser and splitter-coupler design very similar to that of the FY07 "Baseline" system. ${ }^{[2]}$ The laser subassembly is based on a flashlamp pumped, Q-switched design utilizing a Nd:Cr:GSGG ${ }^{[6]}$ laser rod. A single flashlamp pumps the laser rod in a close-coupled, diffuse reflecting, pump cavity. The multimode resonator design provides an output beam with a center weighted intensity profile well suited for fiber injection. The 2008 Prototype implements two modifications to the laser resonator. A rhomboid prism is placed between the Q-switch crystal and the alignment wedge pair, nearest the fixed porro prism (Figure 2). The rhomboid prism induces a $3.7 \mathrm{~mm}$ offset in the laser beam optical path. Additionally, the Spectralon pump cavity was replaced with a redesigned packed barium sulfate pump cavity. The barium sulfate pump cavity historically has provided better optical pumping efficiency. It was also felt that the redesigned pump cavity increase the mechanical robustness of the laser assembly.

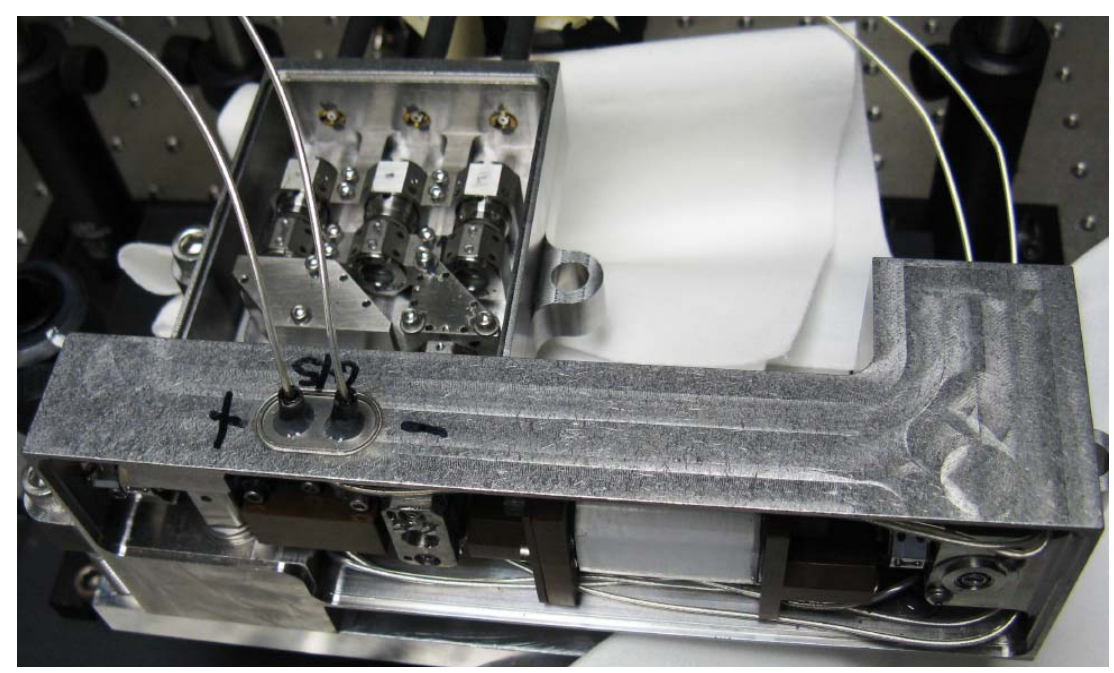

Fig. 1. The 2008 Prototype system, flashlamp pumped, Q-Switched, laser and 3-channel splitter coupler.
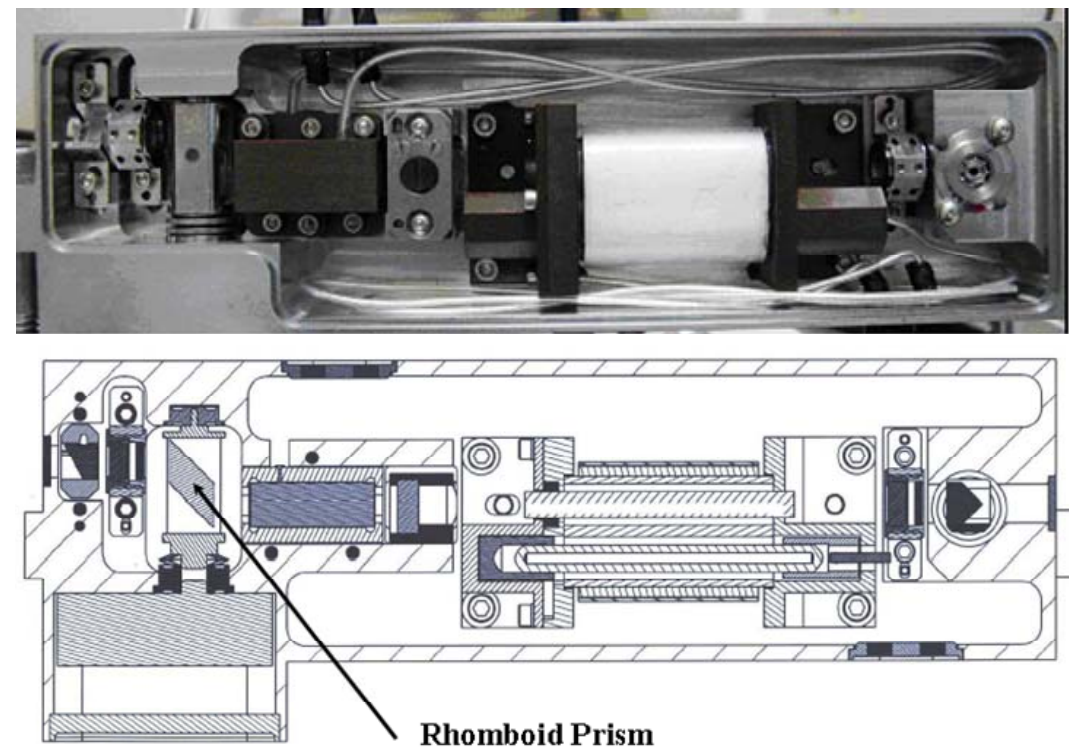

Fig. 2. The 2008 Prototype system’s, flashlamp pumped, Q-Switched, Nd:Cr:GSGG laser. 
The 2008 Prototype system also utilizes a splitter-coupler (SC) design (Figure 3) that is very similar to that of the FY07 "Baseline" system. ${ }^{[2]}$ The SC scheme is based on a straightforward approach utilizing partially reflecting mirrors, placed at 45-degree Angle of Incidence (AOI), to split the incident laser beam into three parallel channels. Three plano-convex lenses focus the beams into the individual optical fibers. Three discrete fiber-optical cables are each terminated with a high power version of a standard SMA-905 connector. The fibers utilized have an inner core diameter of 365 microns $(\mu \mathrm{m})$, with an outer cladding diameter of approximately $400 \mu \mathrm{m}$.
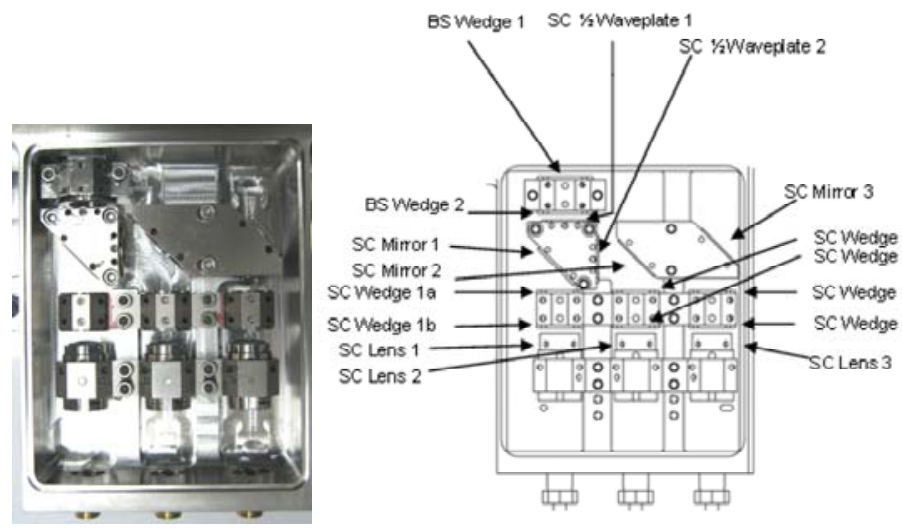

Fig. 3. The three channel splitter-coupler modules for the 2008 Prototype system.

A custom designed power supply and control electronics unit was utilized to power the laser during alignment and characterization testing. The flashlamp Pulse Forming Network (PFN) consists of a high voltage trigger circuit and an Inductor-Capacitor circuit. The flashlamp PFN circuit utilizes a 21.7 micro-farad ( $\mu$ f) capacitor and a 36 micro-henry $(\mu \mathrm{h})$ inductor to provide a critically damped pulse across the flashlamp that is approximately 80 microseconds ( $\mu$ sec) wide at $1 / 3$ peak points. The Q-switch PFN produces a high voltage $(-3 \mathrm{kV})$ pulse across the $\mathrm{LiNbO}_{3} \mathrm{Q}$-switch crystal. The high voltage pulse is flat during the laser pulse build-up time and has a fast fall-time (Figure 4).

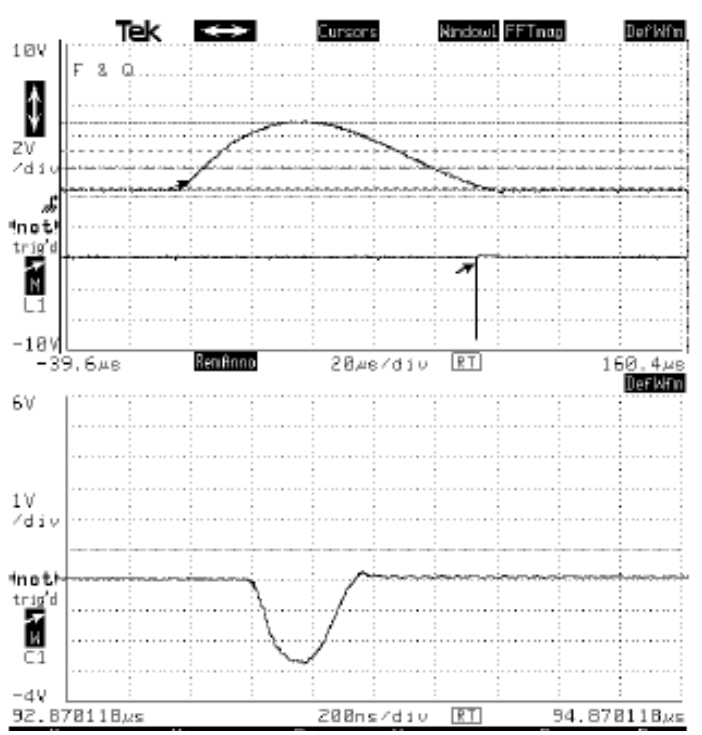

Fig. 4. The flashlamp and Q-switch waveforms generated by the power supplies utilized for the 2008 Prototype system. 


\section{2008 PROTOTYPE PERFORMANCE}

\subsection{Laser Performance}

An acceptable operating goal for the laser module was to obtain between 65 and 80 milli-Joules (mJ) of output energy with a 10 to 13 nanosecond (ns) pulsewidth, measured at the Full Width Half Maximum (FWHM) points. All four prototypes were capable of providing the desired output energy (Figure 5) at an acceptable pulsewidth (Figure 6).

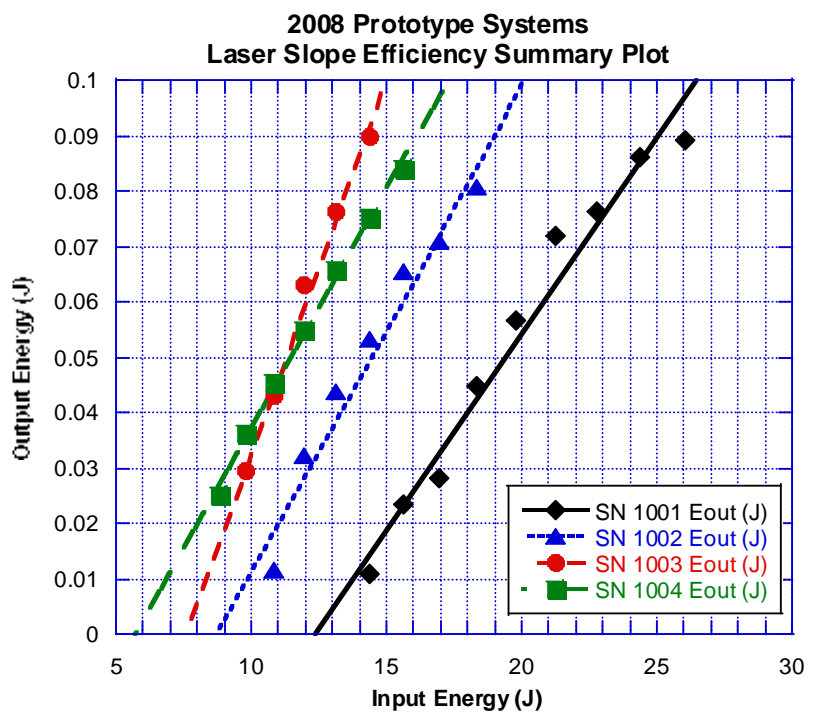

Fig. 5. 2008 Prototypes summary plot: Laser slope efficiency plots as a function of electrical input energy.

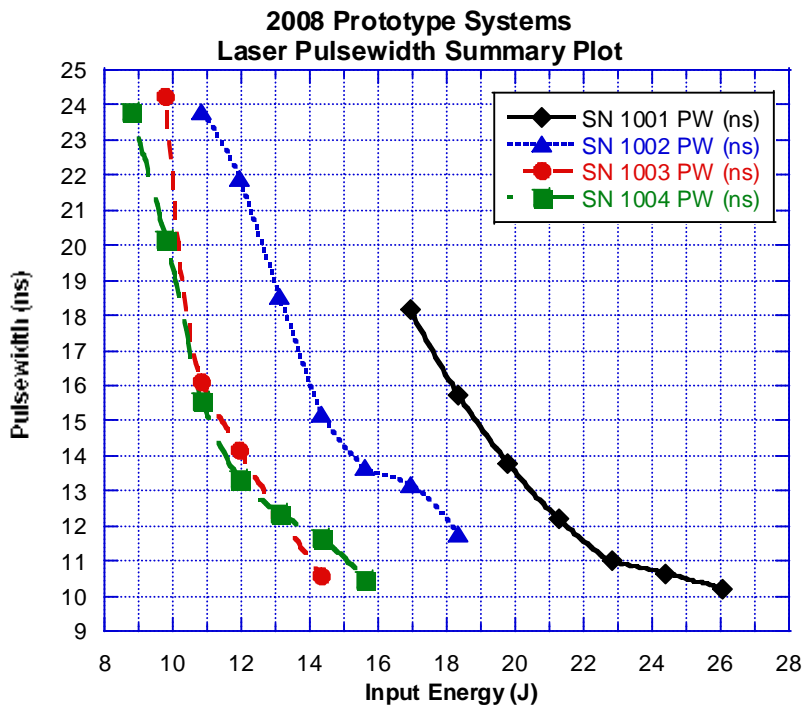

Fig. 6. 2008 Prototypes summary plot: Laser pulsewidth plots as a function of electrical input energy plots. 
The pulsewidths obtained from the four Prototype lasers ranged between 11.4 and $12.5 \mathrm{~ns}$. The laser outputs all had a smooth temporal profile with very little modulation (Figure 7).

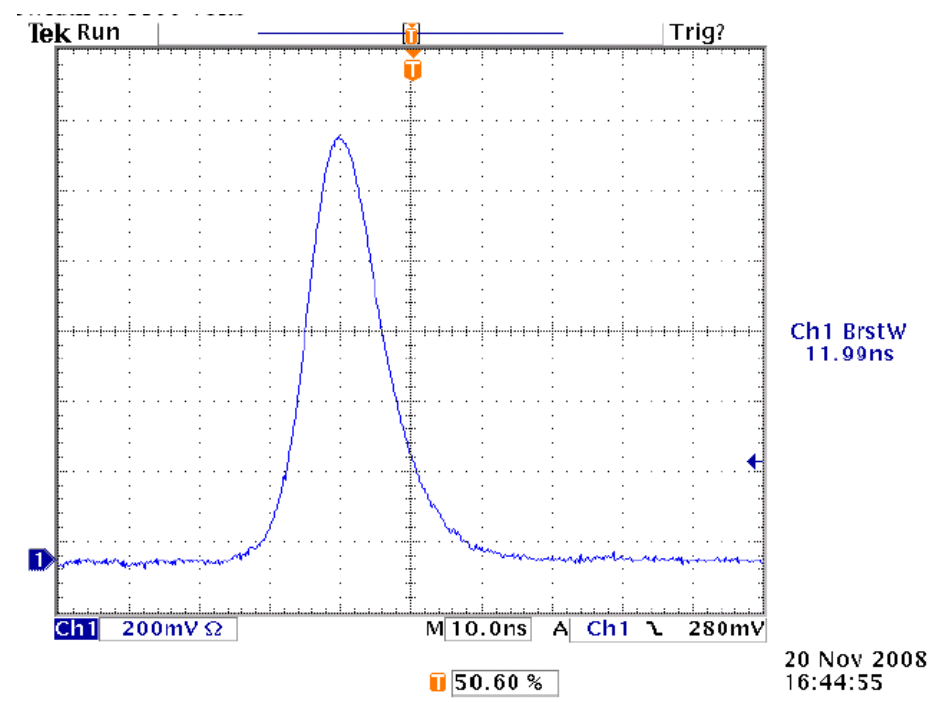

Fig. 7. 2008 Prototypes: Typical laser pulsewidth temporal waveform.

All of the 2008 Prototype lasers were capable of providing the desired output energy and pulsewidth. However, there was a fair amount of variation observed in the electrical input energy required to realize each associated operating point. Table 1 summarizes the slope efficiency and point efficiency associated with the selected operating point of each laser. Laser serial number 1003 had the best performance efficiency; serial number 1001 had the lowest efficiency, while serial numbers 1002 and 1004 performed similarly. The range of efficiencies from lowest to highest was nearly a factor 2.

Table 1. Slope efficiency and point efficiency values for each 2008 Prototypes Laser assembly at selected operating points.

\begin{tabular}{|c|c|c|c|c|}
\hline Laser & Output Energy (mJ) & Pulsewidth (ns) & Slope Efficiency (\%) & Point Efficiency (\%) \\
\hline SN 1001 & 77 & 11.4 & 0.70 & 0.36 \\
\hline SN 1002 & 71 & 12.5 & 0.87 & 0.41 \\
\hline SN 1003 & 76 & 11.9 & 1.34 & 0.58 \\
\hline SN 1004 & 75 & 11.6 & 0.86 & 0.52 \\
\hline
\end{tabular}

All of the 2008 Prototype lasers were capable of providing acceptable spatial intensity profiles and far-field beam divergence. The laser output beams were highly multimode with a relatively low peak-to-average variation across the diameter (Figure 8). The lasers all provided a far-field spatial intensity profile that was smooth and symmetric with a center weighted intensity pattern (Figure 9). The full angle, far-field beam divergence on average was 2.7 milli-radians $(\mathrm{mR})$, when measured at the $90 \%$ points. The beam divergence values and spatial intensity profiles observed are typical of what might be expected from a multimode laser utilizing this type of Polarization Output Coupled (POC) resonator design. 


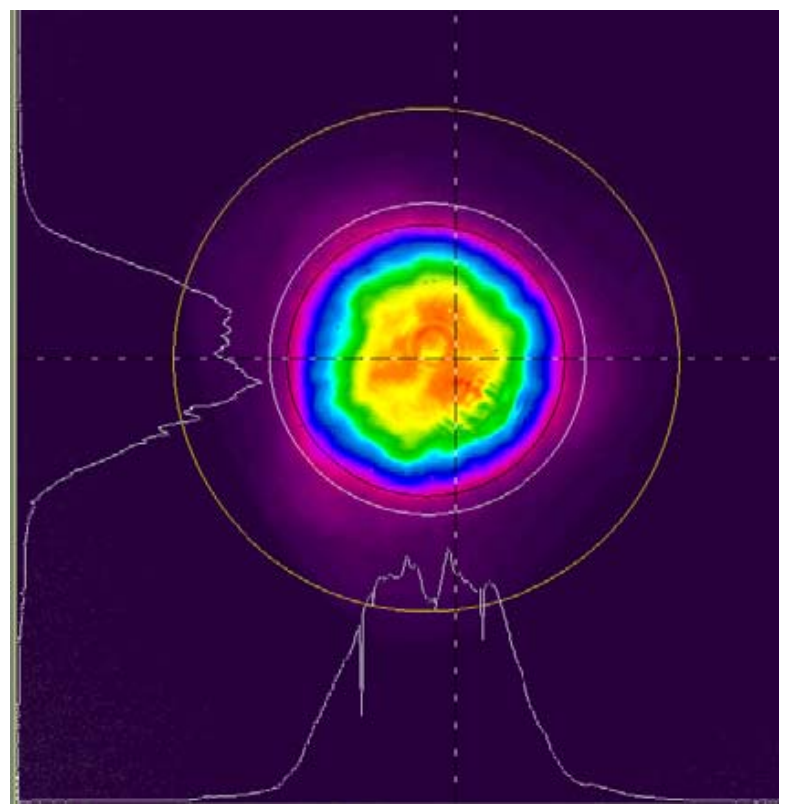

Fig. 8. 2008 Prototype lasers: Representative near-field spatial intensity profile.

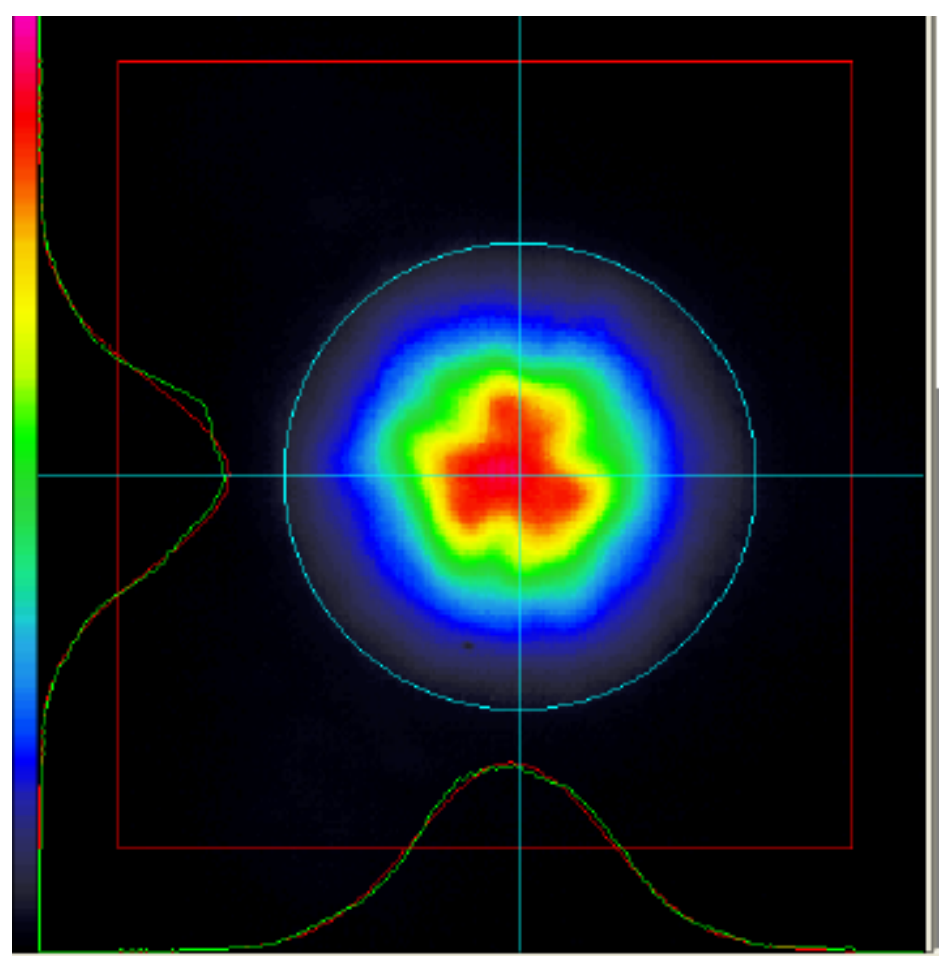

Fig. 9. 2008 Prototype lasers: Representative far-field spatial intensity profile. 
There were some performance variations observed in the intensity profile, the beam divergence and beam diameter measurement. Table 2 summarizes these beam parameters for the prototype lasers, along with their associated mean and standard deviation. Laser serial number 1001 had the lowest beam divergence and lowest peak-to-average intensity variation, while serial number 1002 had the highest beam divergence and highest peak-to-average intensity variation. All of the lasers exhibited a near-field beam diameter that was less than the $5 \mathrm{~mm}$ diameter of the Nd:Cr:GSGG laser rod. Typically when the laser beam does not fully fill the bore diameter of the laser rod, this is an indication of a nonoptimized pumping condition. Serial number 1003 had the largest measured beam diameter, at 4.4mm, and exhibited the best slope efficiency, $1.34 \%$. The degree of variability observed in these beam parameters was considered acceptable. It was not anticipated that any of the 2008 Prototype lasers would have difficulty injecting into the $365 \mu \mathrm{m}$ diameter optical fibers. Initially, it appeared that incorporating the rhomboid prism into the laser resonator design had not affected the performance of the laser module.

Table 2. 2008 Prototypes lasers: Beam divergence, diameter and peak-to-average intensity variation.

\begin{tabular}{|c|c|c|c|}
\hline Laser & $\begin{array}{c}\text { Far-Field Beam } \\
\text { Divergence (mR) }\end{array}$ & $\begin{array}{c}\text { Far-Field Peak-to-Average } \\
\text { Intensity Variation }\end{array}$ & $\begin{array}{c}\text { Near-Field Beam } \\
\text { Diameter (mm) }\end{array}$ \\
\hline SN 1001 & $\mathbf{2 . 2}$ & $\mathbf{2 . 3}$ & 3.9 \\
\hline SN 1002 & $\mathbf{3 . 2}$ & $\mathbf{2 . 6}$ & $\mathbf{3 . 8}$ \\
\hline SN 1003 & $\mathbf{2 . 7}$ & $\mathbf{2 . 4}$ & $\mathbf{4 . 4}$ \\
\hline SN 1004 & $\mathbf{2 . 6}$ & $\mathbf{2 . 5}$ & 3.7 \\
\hline Average & 2.7 & 2.4 & 3.9 \\
\hline $\begin{array}{c}\text { Standard } \\
\text { Deviation }\end{array}$ & $0.41(15 \%)$ & $0.14(8 \%)$ & $0.31(6 \%)$ \\
\hline
\end{tabular}

\subsection{Splitter-Coupler Performance}

The 2008 Prototype system utilizes a SC design that is almost exactly the same as that utilized for the FY07 "Baseline" system. ${ }^{[2]}$ The only modification incorporated into the 2008 SC was a slight redesign to one of the optical mounts to address some beam clipping. This straightforward SC design is well characterized and has a demonstrated ability to provide channel-to-channel split ratios within $\pm 3 \%$. Additionally, the overall efficiency of the SC design is very good, with measured transmission values of between $90-92 \%$ being typical. This is due to the low intrinsic losses associated with the dielectric coated mirrors. Table 3 provides a summary comparison of the SC performance obtained for three of the 2008 Prototype systems. The table compares the split ratios, the high-to-low maximum variation and the deviation from the ideal design goal of 33.3\% splitting per channel.

Table 3. 208 Splitter-Coupler Modules: Detailed performance analysis.

\begin{tabular}{|c|c|c|c|}
\hline \multicolumn{4}{|c|}{ Normalized Split Ratio Percentages } \\
\hline System & SN 1001 & SN 1002 & SN 1003 \\
\hline Channel No. 1 & $32.9 \%$ & $33.9 \%$ & $33.7 \%$ \\
\hline Channel No. 2 & $36.7 \%$ & $34.0 \%$ & $32.4 \%$ \\
\hline Channel No. 3 & $30.4 \%$ & $32.1 \%$ & $33.9 \%$ \\
\hline High-to-Low variations & $6.27 \%$ & $1.98 \%$ & $1.44 \%$ \\
\hline \multicolumn{4}{|c|}{ Deviations from design goal (33.3\%) } \\
\hline Channel No. 1 & $-0.43 \%$ & $0.58 \%$ & $0.40 \%$ \\
\hline Channel No. 2 & $3.35 \%$ & $0.70 \%$ & $-0.92 \%$ \\
\hline Channel No. 3 & $-2.91 \%$ & $-1.28 \%$ & $0.53 \%$ \\
\hline
\end{tabular}


Results are only available for three of the four 2008 SC systems. Due to schedule and budget constraints it was not possible to complete the channel-to-channel split ratio optimization for SN 1004. However, it was possible to characterize the laser-to-fiber alignment and pulse laser injection properties for all four SC modules. The focusing and fiber injection characteristics of the 2008 SC module are also well understood and predictable. The focused spot diameter is solely determined by the laser's beam divergence and the focal length of the injection lens. The fiber utilized is a standard multimode, step index, all fused silica fiber with a numerical aperture (NA) of 0.22. The fiber has an inner core diameter of $365 \mu \mathrm{m}$ and an outer cladding diameter of approximately $400 \mu \mathrm{m}$. Three discrete fiber-optical cables are utilized, each terminated with a high power version of a standard SMA-905 connector. For high peak power laser injection into a $365 \mu \mathrm{m}$ fiber diameter fiber, an incident focus spot corresponding to $80 \%$ of the diameter, approximately $290 \mu \mathrm{m}$, is utilized. This injection geometry, when properly implemented, has demonstrated very good cable-to-cable interchangeability. A similar version of this SC design has been utilized to inject maximum energies of between 40 and $45 \mathrm{~mJ}$ into $400 \mu \mathrm{m}$ diameter fibers. This represents a laser fluence upper limit of approximately $56 \mathrm{~J} / \mathrm{cm}^{2}$. Assuming a similar laser pulsewidth and peak-to-average intensity variation, this would correspond to a maximum upper limit of approximately $37 \mathrm{~mJ}$ for the $365 \mu \mathrm{m}$ diameter fiber. The $2008 \mathrm{SC}$ modules set an operating goal of approximately $25 \mathrm{~mJ}$ per channel. The maximum operating point realized was $27.4 \mathrm{~mJ}$ per channel in system SN 1002 (Table 4). This operating point corresponds to a laser fluence of approximately $41 \mathrm{~J} / \mathrm{cm}^{2}$. Even given this modest safety margin, laser damage has seldom been a problem, assuming the incident beam is well aligned and the optical fiber is clean and defectfree. Past experience has also shown that air breakdown and plasma formation at the intermediate focal plane of the injection lens has seldom been a problem for this robust SC design.

Table 4. Splitter-Coupler SN 1001: Laser fluence incident on entrance face of the $365 \mu \mathrm{m}$ diameter fiber.

\begin{tabular}{|l|c|c|}
\hline \multicolumn{3}{|c|}{ Incident Laser Fluence } \\
\hline & Serial No. 1002 \\
\hline & Energy (mJ) & Fluence $\left(\mathrm{J} / \mathrm{cm}^{2}\right)$ \\
\hline Channel 1 & 27.4 & 40.9 \\
\hline Channel 2 & 27.5 & 41.0 \\
\hline Channel 3 & 25.9 & 38.7 \\
\hline
\end{tabular}

The 2008 SC module provided very clean spatial intensity profiles at the exit faces of the $365 \mu \mathrm{m}$ diameter fibers. The spatial intensity profiles were center weighted and relatively smooth, with small peak-to-average intensity variations of 1.3 or less (Figure 10).
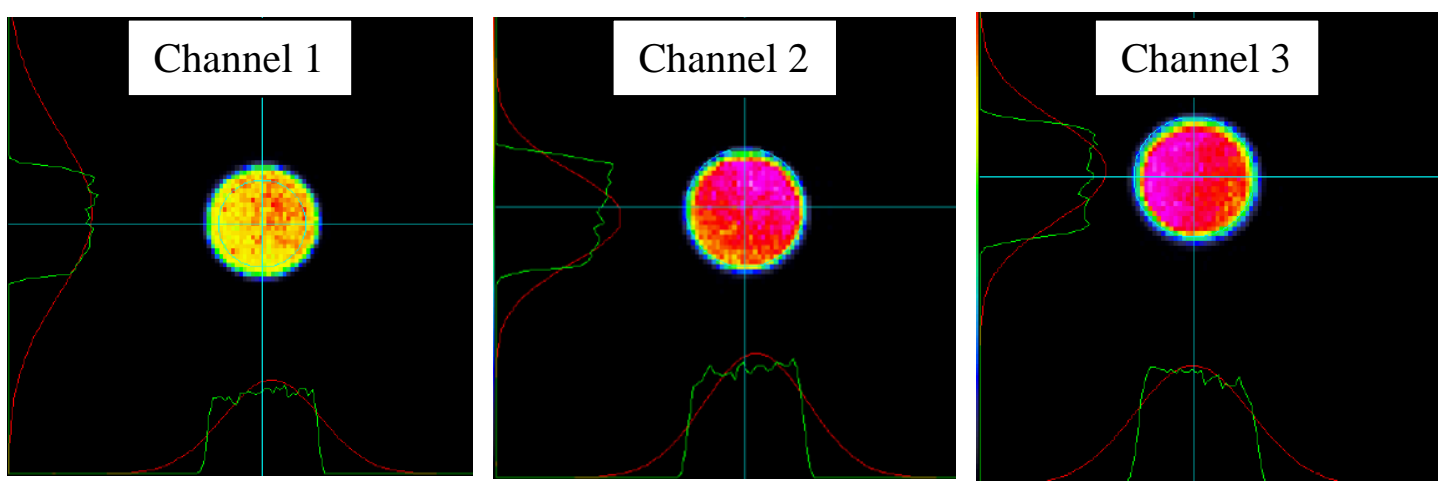

Fig. 10. 2008 Prototype Systems - Typical Spatial Intensity Profile observed at the exit faces of $365 \mu m$ diameter optical fibers. 
During the course of the assembly and characterization of the four 2008 Prototypes, a number of assembly issues were encountered. It was noted that System SN 1001 had a laser module with a considerably lower than expect slope efficiency. Given that several resonator design modifications had been implemented, and that it was unclear what the ultimate ramifications of these changes might be, the unit was moved on. SN 1001 continued to move through the assembly and test process rather uneventfully until the final stages of the SC alignment. An unexpected amount of difficulty was encountered attempting to inject into the $365 \mu \mathrm{m}$ diameter fibers. Even with superior laser-to-fiber alignment, fiber entrance face and connect related laser damage were repeatedly encountered. After an extensive amount of troubleshooting it was found that numerous satellite spots were present at the fiber injection plane (Figure 11). This made it impossible to inject into the $365 \mu \mathrm{m}$ diameter fiber optical cables. Given schedule constraints, it was not possible at the time to determine the root cause of the satellite spots. In place of the $365 \mu \mathrm{m}$ diameter cable, an 800 -to- $400 \mu \mathrm{m}$ diameter optical taper was utilized. The larger $800 \mu \mathrm{m}$ entrance face accepted the incident beam with out suffering laser damage. The larger bend radius associated with the thicker fiber posed a real problem however and it was clear that this would not provide a lasting solution.

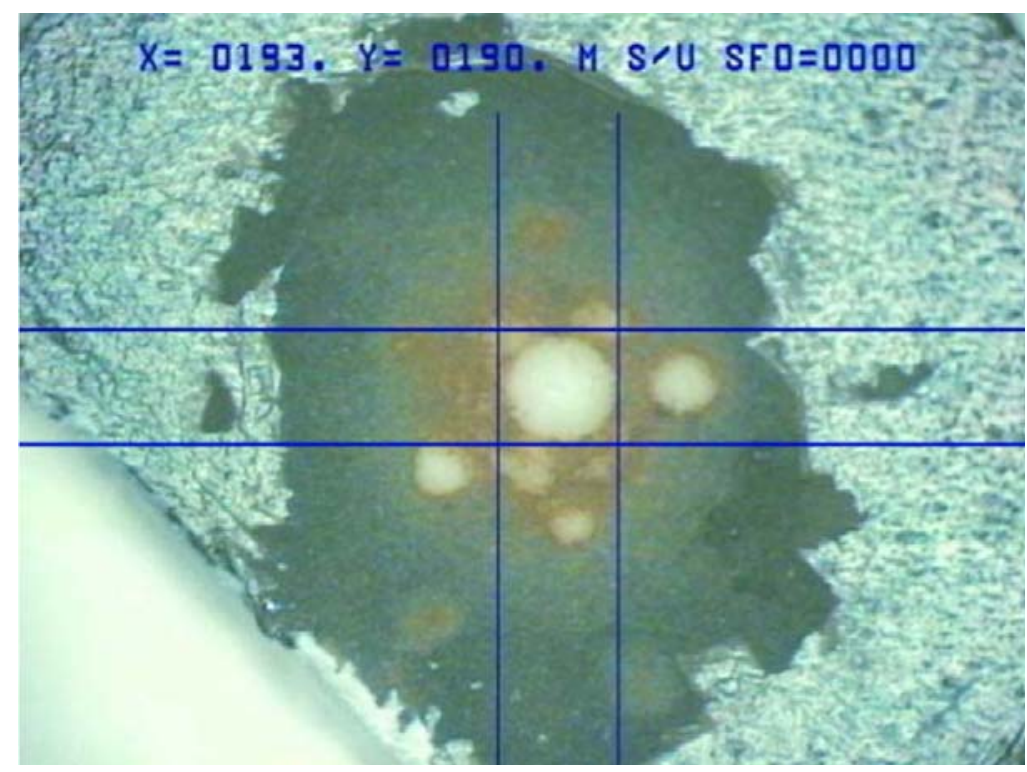

Fig. 11. 2008 Prototype System SN 1001: Satellite spots observed at the entrance faces of the $365 \mu \mathrm{m}$ diameter optical fiber.

During the very early assembly stages of laser SN 1002, it was noted that the Nd:Cr:GSGG laser rods ordered to support the 2008 hardware build were of a markedly different color. When compared to laser rods received on previous orders, these laser rods were a lighter shade of green. The decision was made to discontinue utilizing the laser rods from this order. Instead, Nd:Cr:GSGG laser rods pulled from inventory were utilized to complete the 2008 Prototypes. Ultimately, it was verified by the supplier that the Nd:Cr:GSGG laser rods delivered on the 2008 order did not have the correct Chromium ion concentration levels. The measured concentration levels were half of that specified on the drawing. The lower than desired Chromium concentration was assumed to be the reason for the poor laser slope efficiency obtained for prototype SN 1001.

Laser module SN 1002 had a much better slope efficiency than SN 1001. It appeared that the laser rod problem had been correctly identified. System SN 1002 moved through the assembly and test process rather uneventfully to the final stages of the SC alignment. At this stage, an exhaustive effort was undertaken to look for satellite spots which might be present in the fiber injection plane. The focused laser spot was not nearly as fragmented as SN 1001 had been. A single satellite spot was found. This spot was present in all three of the SC channels (Figure 12). The spots were located at a distance of approximately $360 \mu \mathrm{m}$ outside of the main beam. However, it was possible to successfully inject into the $365 \mu \mathrm{m}$ diameter cables. 


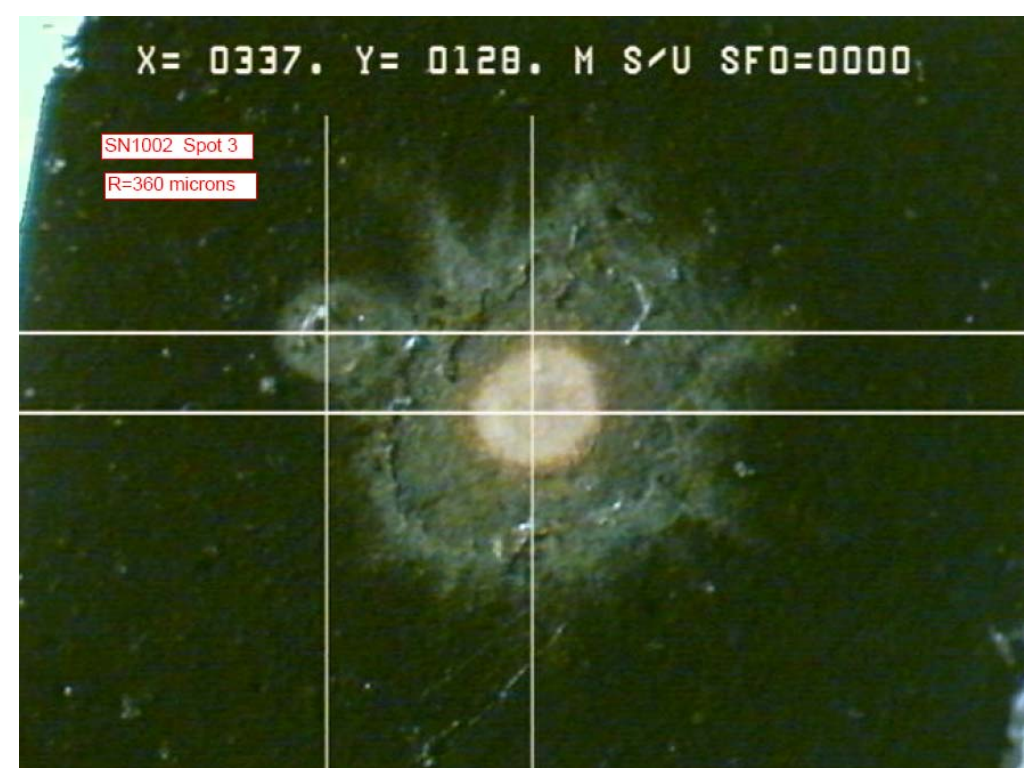

Fig. 12. 2008 Prototype System SN 1002: Satellite spots observed at a distance of approximately 360 $\mu$ m outside of the main beam.

System SN 1003 had a laser module with the highest slope efficiency, 1.34\%, of all the 2008 laser modules. SN 1003 moved through the assembly and test process to the final stages of the SC alignment uneventfully. Once again, an exhaustive effort was undertaken to look for satellite spots which might be present in the fiber injection plane. The focused laser spot was much cleaner than any of the previous systems. A single satellite spot, of weak intensity was found, but only in channel number 1 of the SC (Figure 13). This spot was determined to be approximately 300 $\mu$ m or more outside of the main beam. Once again, it was possible to successfully inject into the $365 \mu$ m diameter cables.

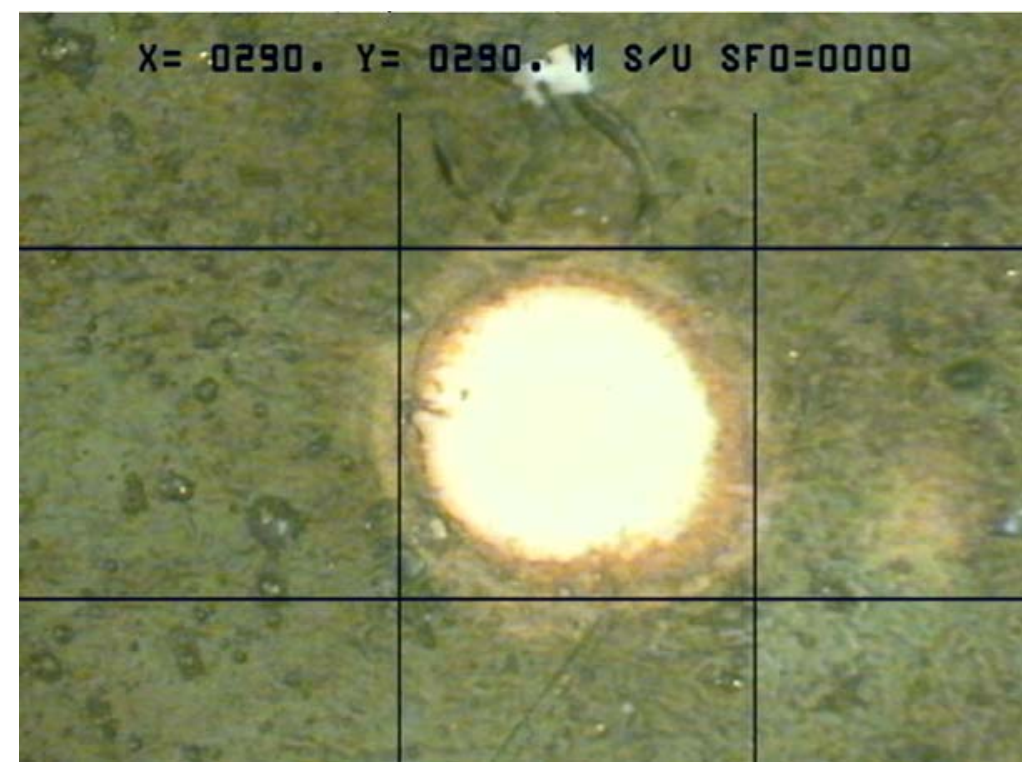

Fig. 13. 2008 Prototype System SN 1003: Satellite spots observed at a distance of approximately 300 $\mu$ m outside of the main beam. 
However, there were only three optical cables remaining. It was necessary to utilize two optical cables that were considered to be out of specification. Variations in the fiber-to-connector ferule concentricity had been measured on several of the optical cables terminated with the high power SMA905 connectors. Typically the concentricity was measured at between 15 and 35 $\mu \mathrm{m}$. Two of the cables, SN 9 and SN 12, were measured to be out of tolerance by 75 and $100 \mu \mathrm{m}$ respectively (Figure 14). A hardware based alignment error of this amount consumes a large portion of the overall tolerance budget for the optical alignment of the system. It was a concern that the use of these cables might compromise the ability of System SN 1003 to endure mechanical environments and maintain adequate fiber alignment.

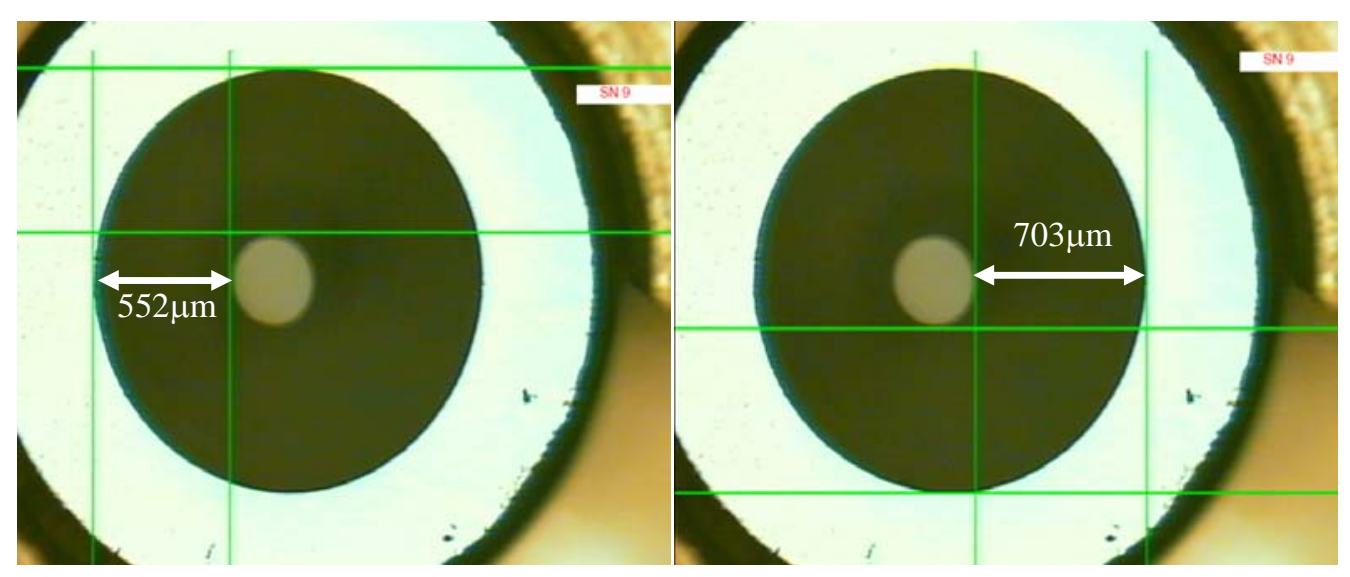

Fig. 14. Fiber optical cable terminated with high power SMA-905.

System SN 1004 had a laser module with a slope efficiency of $0.86 \%$, approximately equal to that of laser module SN 1002. SN 1004 move through the assembly and test process to the final stages of the SC alignment without difficulty. However, before work could begin to document the presence of any satellite spots, the project funding ran out. A much less rigorous investigation was undertaken. Due to the funding and schedule related difficulties, SC module SN 1004 was not fully optimized. The laser-to-fiber alignment and injection was completed, however it was not possible to complete the channel-to-channel energy balancing. Unfortunately, the available inventory of high power SMA-905 terminated fiber optical cable, with the $365 \mu \mathrm{m}$ diameter was exhausted. Prototype SN 1004 was successfully test fired on several instances into a surrogate optical cable having a diameter of $400 \mu \mathrm{m}$.

\section{SUMMARY}

The assembly and characterization of the latest design iteration for a small, ruggedized laser-optical firing system was completed. Four identical prototypes were assembled and characterized to obtain a sense of the assembly and performance repeatability of this design. The performance of the units was evaluated to compare and contrast a number of important parameters including laser output energy, slope efficiency, beam divergence, spatial intensity profile, fiber injection and splitter-coupler transmission efficiency. One area of improvement investigated, was the implementation of a second optical safing and arming component. A rhomboid prism was incorporated into the laser resonator for this purpose. The rhomboid prism did not appear to negatively impact the laser performance itself, however this optic can not be ruled out as one possible source of the satellite spots present in the fiber injection plane. 


\section{FUTURE WORK}

Future plans for the continued test and evaluation of this design have been put on hold by Sandia National Laboratories. The desire would be to support continued environmental testing and design optimization activities as appropriate. Additional investigation is required to determine the root cause for the observed satellite spots. Longer term plans are still concerned with developing a well defined strategy to hermetically seal the entire laser-optical system. A combined approach is being considered which would make use of sealed windows, hermetic fiber optical and electrical connectors and welded covers.

\section{ACKNOWLEDGMENTS}

This work was supported by the U.S. Department of Energy under contract DE-AC04-01AL66850. Project specific funding was provided by Sandia National Laboratories. The authors would like to recognize and thank Tanner Vaughn for his work in the laboratory bonding, aligning and characterizing the numerous optical assemblies, lasers and SC systems. Additionally, Dante Berry of Sandia National Laboratories must be recognized for his painstaking efforts at fabricating the fiber-optical cables.

\section{REFERENCES}

[1] G. Morelli, "A Comparison of Two Laser-Optical Firing Systems", Proceedings of SPIE Vol.7070, p. 6-1, Aug. 2008.

[2] G. Morelli, "Design and Assembly Strategies for Two Laser-Optical Firing Systems", Proceedings of SPIE Vol.6662, p. 1, Aug. 2007.

[3] M. Bright, G. Morelli, “Characterization of Optical Components for use in Harsh Environments”, SPIE Proc 62873, 2006.

[4] M. Bright, G. Morelli, “Characterization of Laser-Optical Systems for use in Harsh Environments”, SPIE Proc 6662-4, 2007.

[5] M. Bright "Post mortem results of laser-optical system packaged for use in harsh environments", SPIE Proc 707014, 2008.

[6] W. Koechner, Solid-State Laser Engineering, $5^{\text {th }}$ Edition, Springer-Verlag Berlin Heidlberg, pp. pp. 57, 1999. 